\title{
COMPARISON OF UAS-BASED PHOTOGRAMMETRY SOFTWARE FOR 3D POINT CLOUD GENERATION: A SURVEY OVER A HISTORICAL SITE
}

\author{
F. Alidoost, H. Arefi * \\ School of Surveying and Geospatial Engineering, College of Engineering, University of Tehran, Tehran, Iran - (falidoost, \\ hossein.arefi)@ut.ac.ir
}

KEY WORDS: 3D Modelling, DSM, Point Cloud, Photogrammetry, Accuracy Assessment

\begin{abstract}
:
Nowadays, Unmanned Aerial System (UAS)-based photogrammetry offers an affordable, fast and effective approach to real-time acquisition of high resolution geospatial information and automatic 3D modelling of objects for numerous applications such as topography mapping, 3D city modelling, orthophoto generation, and cultural heritages preservation. In this paper, the capability of four different state-of-the-art software packages as 3DSurvey, Agisoft Photoscan, Pix4Dmapper Pro and SURE is examined to generate high density point cloud as well as a Digital Surface Model (DSM) over a historical site. The main steps of this study are including: image acquisition, point cloud generation, and accuracy assessment. The overlapping images are first captured using a quadcopter and next are processed by different software to generate point clouds and DSMs. In order to evaluate the accuracy and quality of point clouds and DSMs, both visual and geometric assessments are carry out and the comparison results are reported.
\end{abstract}

\section{INTRODUCTION}

Recent developments in the UAS-based data acquisition techniques and low cost aerial imaging sensors improve the speed of rapid 3D mapping in numerous civil, agriculture, military and archeology applications. In order to process resulting several hundred high resolution overlapping images and therefore automatic $3 \mathrm{D}$ reconstruction, different photogrammetry and computer vision algorithms have been developed based on dense image matching techniques. These algorithms first extract the corresponding features or points in the overlapping images and then compute 3D information for each pixel. Currently, a number of commercial and open source tools and software are available to handle all steps of an image based 3D modeling framework from image preprocessing to generation of 3D geospatial products like point clouds, DSMs, orthophotos, mesh models, texture models and so on. Since the selection of best solution for $3 \mathrm{D}$ modeling and mapping is an important issue in many projects, the performance of the state of the art UAS-based photogrammetric software are evaluated in current researches (Schwind and Starek, 2017; Burns and Delparte, 2017; Svensk, 2017; Zečević, et al., 2017; Niederheiser, et al., 2016; Haala and Cavegn, 2016; Bhandari, et al., 2015; Remondino, et al., 2014; Haala, 2013). Two well-known commercial software, i.e. Pix4D and Agisoft Photoscan, as well as the OpenDroneMap as an open source software are compared based on the quantity and quality parameters over varying terrain. Based on the results of this assessment, the total number of generated points by Pix4D is higher than other software. However, the accuracies of point cloud generated by Pix4D and Agisoft Photoscan are very close to each other in different types of land covers and higher than the accuracy of point cloud generated by OpenDroneMap (Schwind and Starek, 2017). In an interesting application, both Pix4D and Agisoft Photoscan software are employed to generate a 3D model of underwater objects with different spatial scales. Three metrics as the image alignment, total spatial error, and reprojection error were used to compare the capability of both software in 3D modeling of complex structures under water. As the result, the image alignment by Agisoft Photoscan is carried out better while the total error of 3D model generated by Pix 4D is lower (Burns and Delparte, 2017). In forest inventory application, four software of Keystone, SURE, Agisoft Photoscan, and MicMac are used to generate point clouds in order to estimate the parameters of trees. Based on the calculated RMSE values, Keystone and SURE performed somewhat better while MicMac placed third and Agisoft Photoscan achieved the less accurate result (Svensk, 2017). In order to produce 3D model of a vegetated rock face, different software packages such as Agisoft PhotoScan, Pix4D, a combination of Visual SFM and SURE, and MicMac are utilized and compared based on visual assessments and height profiles. In this evaluation, acquired terrestrial images by compact cameras with different sensor sizes and lenses and default parameters of software are used to generate point clouds. In this application, the generated point clouds by Agisoft PhotoScan and MicMac have better accuracy (Niederheiser, et al., 2016). In another study, the generated DSM by Agisoft PhotoScan, Pix4D and LPS over a sand mine are compared using the ground control points. Based on the results, the RMSE and mean error of Pix4D's DSM is lower than other two software. According to accuracy of generated DSM by LPS, it seems that using an aerial image-based software is not suitable for processing the UAV-based images (Bhandari, et al., 2015). A visual comparison between point clouds generated by different commercial and open source dense image-matching software such as SURE, MicMac, Agisoft PhotoScan and PMVS is carry out for both close range and aerial imaging applications. Based on the height profiles, the performance of software to generate point clouds depends on the type of application and the terrain. Also, a clear ranking of software is not reported. However, Agisoft PhotoScan and SURE have more appealing and reliable results (Remondino, et al., 2014). A visual assessment between different DSMs generated by various photogrammetric software such as SURE, MicMac, SocetSet, Match-T and the German Aerospace Center (DLR) matching algorithm is carry out on a benchmark dataset from the European Spatial Data Research Organization (EuroSDR). For better evaluation of image matching algorithms, the height profiles on different land covers

\footnotetext{
* Corresponding author
} 
such as building, river, trees and shadows are compared (Haala, 2013).

\section{MATERIALS AND METHOD}

In this study, the most commonly used UAS-based photogrammetry software packages are selected to generate 3D models of a historical site and the results are then compared. The main steps of this study as shown in Figure 1 are including: image acquisition, point cloud generation, and accuracy assessments. In the first step, about 300 aerial photos with $70-80 \%$ overlap and $0.97 \mathrm{~cm}$ spatial resolution were taken at a flying altitude of about 40 m over the ancient city of Harireh (Wikipedia, 2017), in Kish Island, Iran (Figure 2). The aerial sensor is a 20-megapixel camera which is installed on a DJI Phantom 4 Pro system (DJI, 2017). After removing the low quality images, only 89 images were retained for $3 \mathrm{D}$ modelling.

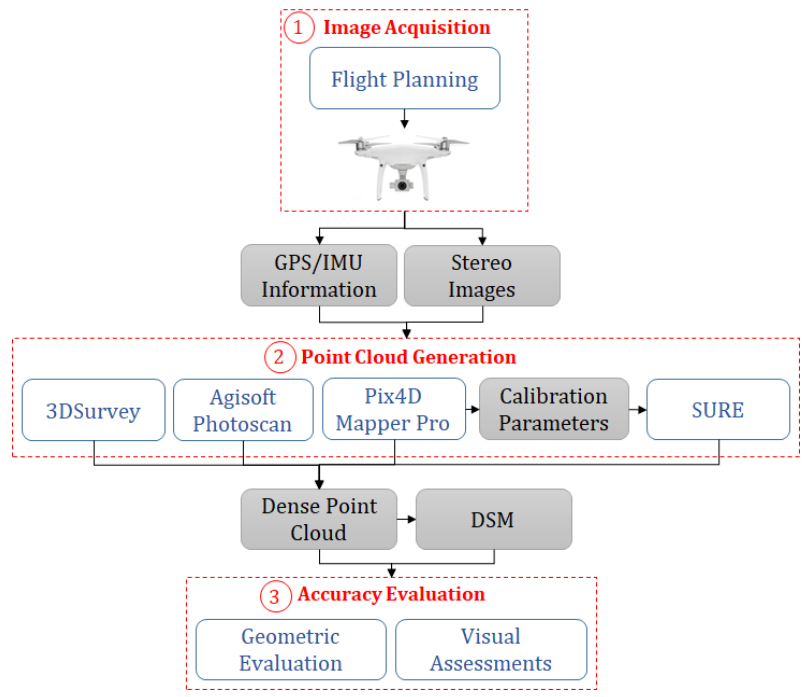

Figure 1. Main flowchart of software comparison

The camera position and orientation parameters are also provided from the on-board Inertial Measurement Unit (IMU) and Global Positioning System (GPS). These parameters are stored as the EXIF data in images' metadata and employed by software for geo-referencing application. Next, the trial versions of UASbased software such as 3DSurvey (3DSurvey, 2017), Agisoft Photoscan (Agisoft Photoscan, 2017), Pix4Dmapper Pro (Pix4D, 2017), and SURE (nFrame, 2017) are utilized for image-based modelling. The common workflow for dense point cloud generation includes pose estimation, image alignment, tie points generation, dense point cloud generation by dense stereo matching techniques such as the Semi-Global Matching (SGM) algorithm (Hirschmueller, 2005), and 3D modelling (Alidoost and Arefi, 2015). The recommended parameters as well as same settings are used to generate dense point clouds (e.g. the "high" option for dense point cloud generation). Moreover, in order to apply same settings, the resulting dense point clouds are just considered as the final output of each software and further used to generate DSM by a third-party software. DSMs with spatial resolution of $2 \mathrm{~cm}$ are generated from point clouds based on the Inverse Distance Weighting (IDW) interpolation technique. Since the image alignment parameters are not calculated by SURE software, orientation information should be imported from the other tools such as VisualSFM, Pix4D, and so on (SURE Manual, 2014). In this study, the "calibrated camera parameters' file" calculated by Pix4D is employed. Moreover, in order to generate a point cloud in a correct global coordinate system (e.g. UTM) by SURE, another file of Pix4D's outputs which it is called the "calibrated external camera parameters" should be in a same folder with the calibrated camera parameters' file to be taken into account as well by SURE (SURE Manual, 2014) automatically. Finally, the geometric accuracy of generated point clouds as well as DSMs are evaluated separately and also to each other using the standard descriptors and the robust descriptors to outliers. In addition, the visual qualities are illustrated by Quantile- Quantile (Q-Q) plots (Höhle, 2009), elevation profiles and error classification maps. If there is a strong deviation from a straight line in Q-Q plots, there is a non-normal distribution of elevation errors because of outliers and some systematic errors in data.

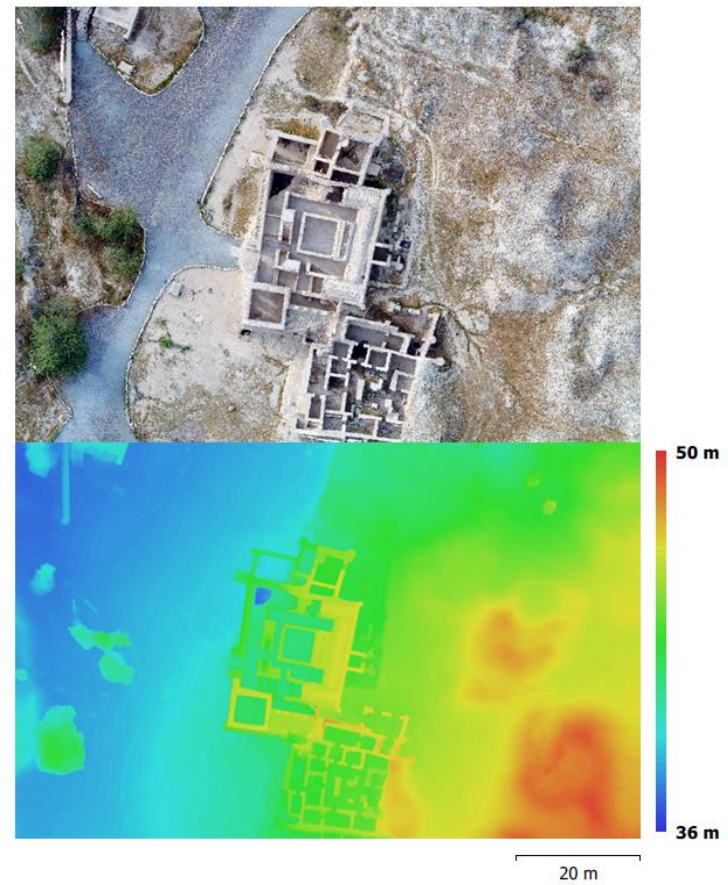

Figure 2. City of Harireh, Kish Island, Iran: Orthophoto (up), DSM (down)

\section{RESULTS AND DISCUSSION}

The software's assessments focused on two main components: visual quality and geometric accuracy of each point cloud as well as two relative accuracy between two point clouds.

\subsection{Absolute Point Cloud Assessments}

In order to evaluate the performance of dense point cloud generation, the number of key points per image, the number of final dense points, point count in a same extent, point spacing, spatial error for horizontal and vertical components, and the computational time are reported as shown in Table 1 . The number of extracted key points per image for Pix4Dmapper Pro is higher while the number of final dense points for Agisoft Photoscan is higher than the other tools. The point cloud of 3DSurvey is less dense, consequently its computational time is higher than the others. The point spacing for point clouds of Agisoft Photoscan and Pix4Dmapper Pro is similar $(0.016 \mathrm{~m})$ and lower than point spacing for two other software. There is no significant difference in spatial errors for point clouds of 3DSurvey and Agisoft Photoscan. In addition, the processing time of Agisoft Photoscan, Pix4Dmapper Pro and SURE is similar to each other, roughly. 
The overall visual quality of resulting 3D point cloud over various land cover types such as walls, hills and trees are shown in Table 2 for each software. It could be concluded that the differences in the total number of points by four software do not indicate significant quality differences in the final 3D reconstruction. For all software, due to different error sources in matching process, the gap areas are created and therefore, the quality of $3 \mathrm{D}$ reconstruction is decreased. For example, because of hidden areas around the trees and mismatching error in vegetated areas as well as shadows in the walls' corners, there are some gaps in generated point clouds. However, the error is low in case of Pix4Dmapper Pro and SURE. In addition, there are some bigger gap areas in walls due to unsuitable view angle of images for all software. Also, the error regarding to the detail missing in the sandy hills due to smoothing processing is clear in the generated point cloud by 3DSurvey.

In order to illustrate the quality of DSMs, several elevation profiles are extracted along different land cover types such as large steep edges (Figure 3), flat surfaces (Figure 4), trees (Figure 5), hills (Figure 6), and small edges (Figure 7). For better evaluation, the elevation errors are categorized into four groups as: smoothing effect (Error type 1: E1), vertical shifts (Error type 2: E2), horizontal shifts (Error type 3: E3), and elevation spikes (Error type 4: E4).

For the 3DSurvey's DSM, there are smoothing and details removing errors specially at the natural topographic objects such as hills (Figures 3, 5, and 6), a large vertical shift (Figure 4), a large horizontal shift (Figure 1), and significant elevation spikes for all land cover types (Figures 3, 4 and 7). For the Pix4Dmapper Pro's and the SURE's DSMs, the results are more appealing and the trend of elevation profiles are similar to each other. Although, the smoothing level in the SURE results is a little more than Pix4Dmapper Pro's results and it has caused the small elevation differences (e.g. appear as a kind of noise) are lower at the continuous surfaces (Figures 3, 4, 5 and 6). Finally, for the Agisoft Photoscan's DSM, there are a significant vertical shift (Figures 3-7), and elevation spikes at the flat surfaces (Figure 4). However, the small elevation differences are smaller for the Agisoft Photoscan's DSM than for the Pix4Dmapper Pro's DSM (Figures 1, 5, and 6). In vegetated areas, it is accepted to have many small height differences at the crowns. However, of the four software, the extracted details by 3DSurvey is lower (Figure $5)$.

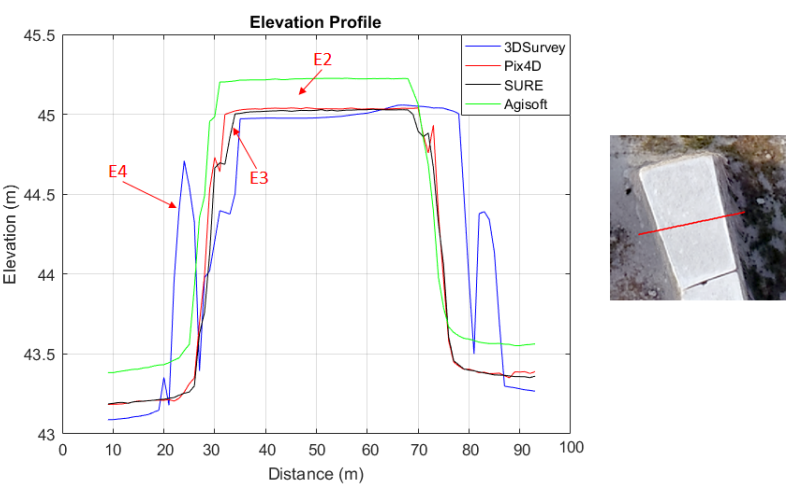

Figure 3. Elevation profiles on DSMs at steep edges

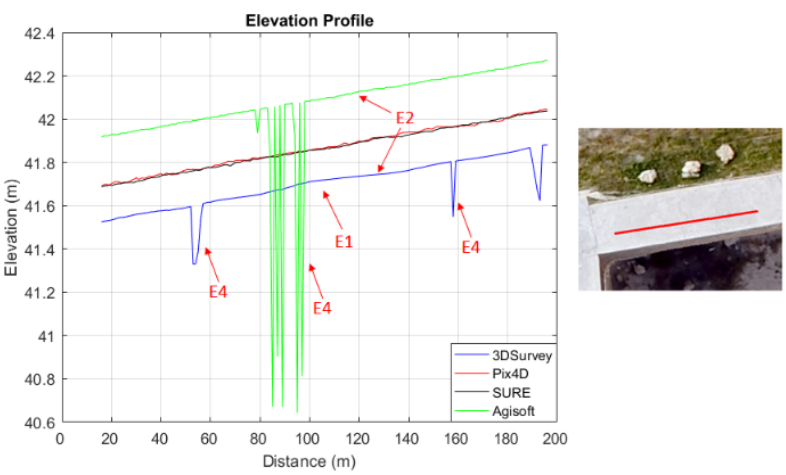

Figure 4. Elevation profiles on DSMs at sloped flat surface

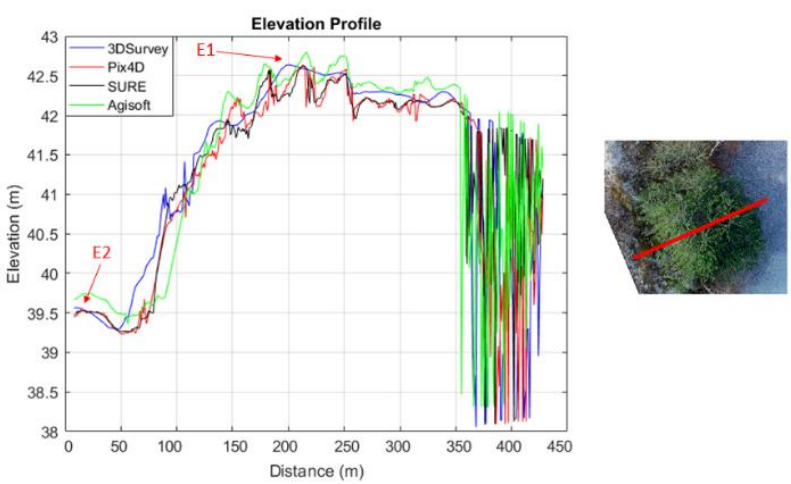

Figure 5. Elevation profiles on DSMs along trees

\begin{tabular}{|l|c|c|c|c|c|c|c|c|}
\hline Software & $\begin{array}{c}\text { Number of } \\
\text { key points } \\
\text { per images }\end{array}$ & $\begin{array}{c}\text { Number } \\
\text { of dense } \\
\text { points }\end{array}$ & $\begin{array}{c}\text { Point count } \\
\text { in a same } \\
\text { extent }\end{array}$ & \multicolumn{2}{|c|}{$\begin{array}{c}\text { Point } \\
\text { spacing } \\
(\mathrm{m})\end{array}$} & \multicolumn{2}{|c|}{$\begin{array}{c}\text { Spatial errors (m) for } \\
\text { X, Y, Z }\end{array}$} & $\begin{array}{c}\text { Computational } \\
\text { time (hour) }\end{array}$ \\
\hline 3DSurvey & 19095 & 2167305 & $1,830,149$ & 0.059 & 0.365 & 0.614 & 0.175 & 10 \\
Agisoft Photoscan & 23070 & 31555687 & $24,679,098$ & 0.016 & 0.346 & 0.691 & 0.209 & 7 \\
Pix4Dmapper Pro & 66168 & 28956540 & $23,954,697$ & 0.016 & 0.386 & 0.386 & 0.929 & 5 \\
SURE & Not reported & 16243685 & $13,620,695$ & 0.021 & \multicolumn{2}{|c|}{ Not reported } & 6 \\
\hline
\end{tabular}

Table 1. Characteristics of generated point clouds by different software 



Point cloud Agisoft Photoscan
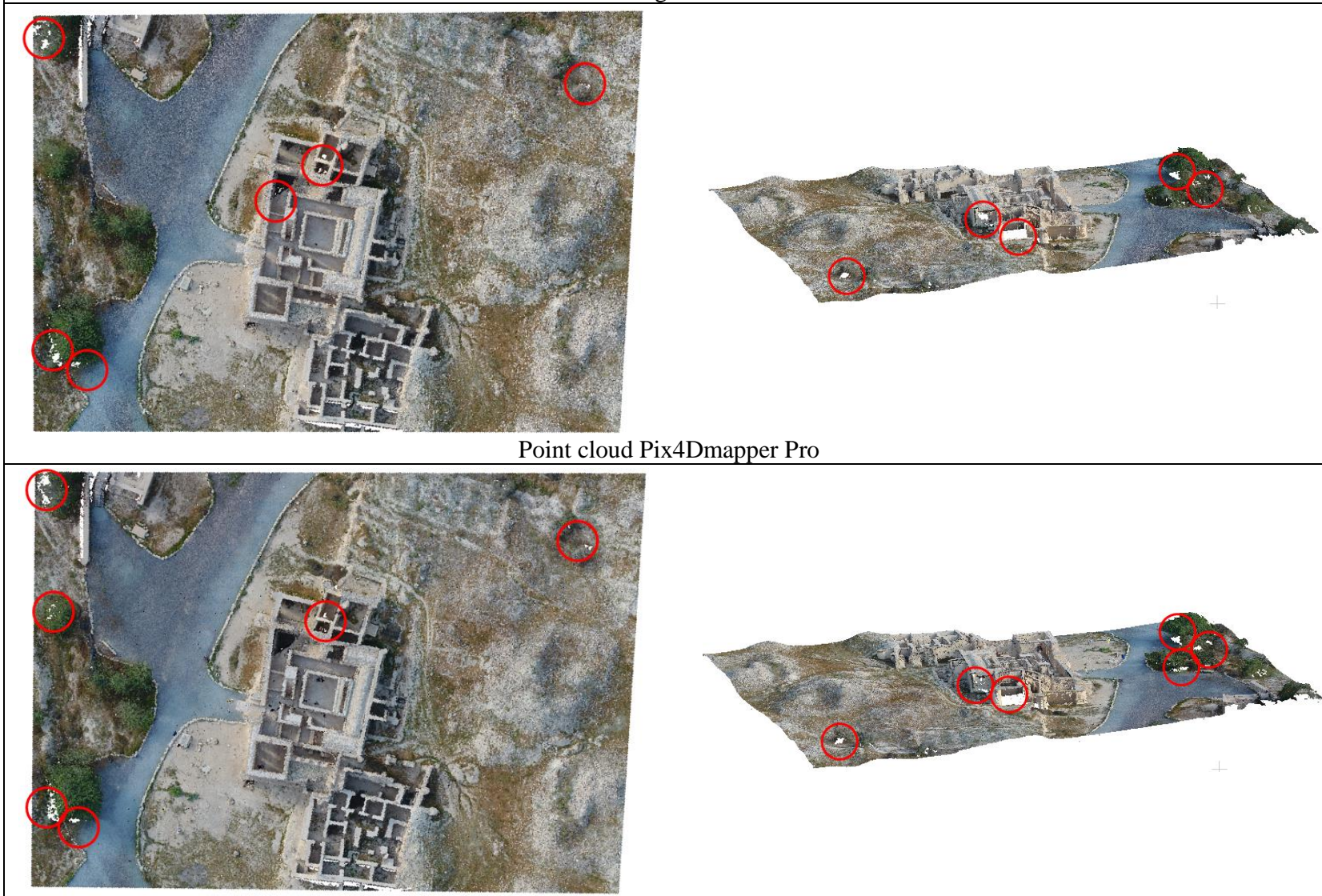

Point cloud SURE

Table 2. Quality of point cloud over different land cover types 


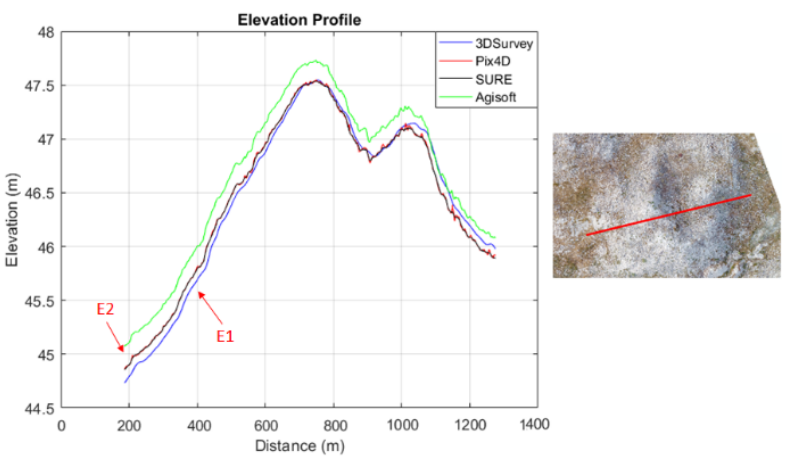

Figure 6. Elevation profiles on DSMs at hills

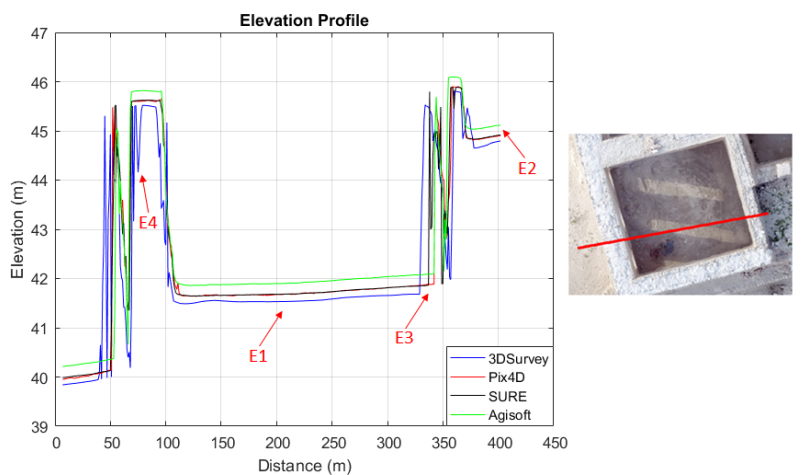

Figure 7. Elevation profiles on DSMs at edges

\subsection{Relative Point Cloud Assessments}

For relative software comparison, the mean error and standard deviation of $3 \mathrm{D}$ distances between point clouds are calculated based on a quadric function included six parameters. As shown in Figure 8, the lowest values of mean error and standard deviation of distances are obtained for point clouds generated by Pix4Dmapper Pro and SURE. This might be because of that SURE was initialized using calculated calibration parameters by Pix4Dmapper Pro. Also, there are a considerable difference between two point clouds generated by Agisoft Photoscan and 3DSurvay due to significant difference in the point densities as well as high level smoothing of points applied by 3DSurvay.

The accuracy assessment of the height differences between two DSMs can be calculated based on standard descriptors such as the mean of errors, Root Mean Square Error (RMSE), Standard Deviation (SD) (Figure 9) and the robust descriptors to outliers as the median of errors, Normalized Median Absolute Deviation (NMAD), and sample quantiles of errors (Höhle, 2009) (Figure 10). According to the mean error value between Agisoft Photoscan's DSM and DSMs of other three software, it could be concluded that there is a significant elevation bias of approximately $0.2 \mathrm{~m}$ in Agisoft Photoscan's DSM. The average of median errors between Agisoft Photoscan's DSM and DSMs of other three software is about $0.25 \mathrm{~m}$ which is a robust measure to prove the existence of systematic vertical shift between the two groups of data.

The RMSE metric is a widely employed measure of conformity between two DSMs, and if the RMSE and SD values are similar, it could be concluded that the distribution of errors is normal and there is no outliers or systematic errors in two DSMs. The lowest values of RMSE and SD $(0.2 \mathrm{~m})$ are for height differences between Pix4Dmapper Pro's and SURE's DSMs, while the highest values of these metrics are for height differences between Agisoft Photoscan's and 3DSurvey's DSMs $(0.4 \mathrm{~m})$. Since the calculated calibration parameters by Pix4Dmapper Pro are used at the initialization step of SURE processing, there is no systematic error (bias) between Pix4Dmapper Pro's and SURE's DSMs and the mean and median errors of height differences is close to zero.

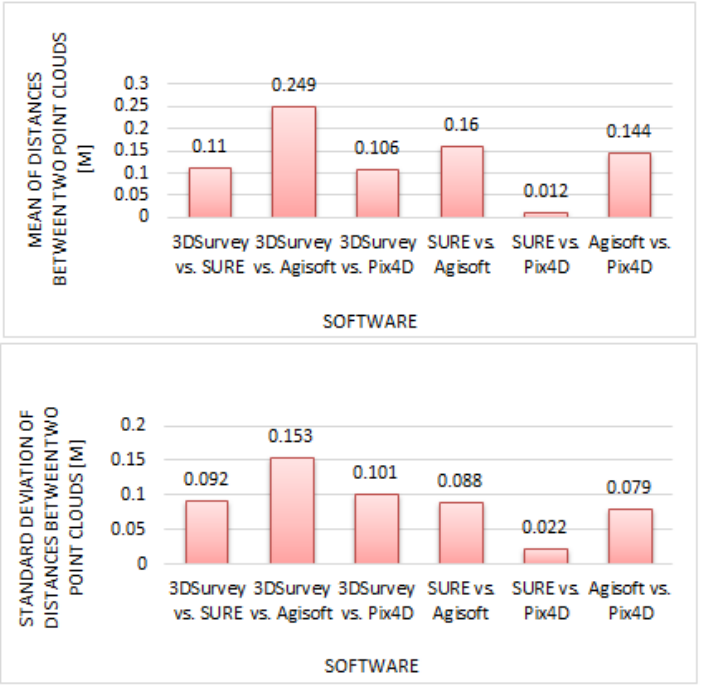

Figure 8. 3D distances [m] between two point clouds: the mean error (up), SD (down)

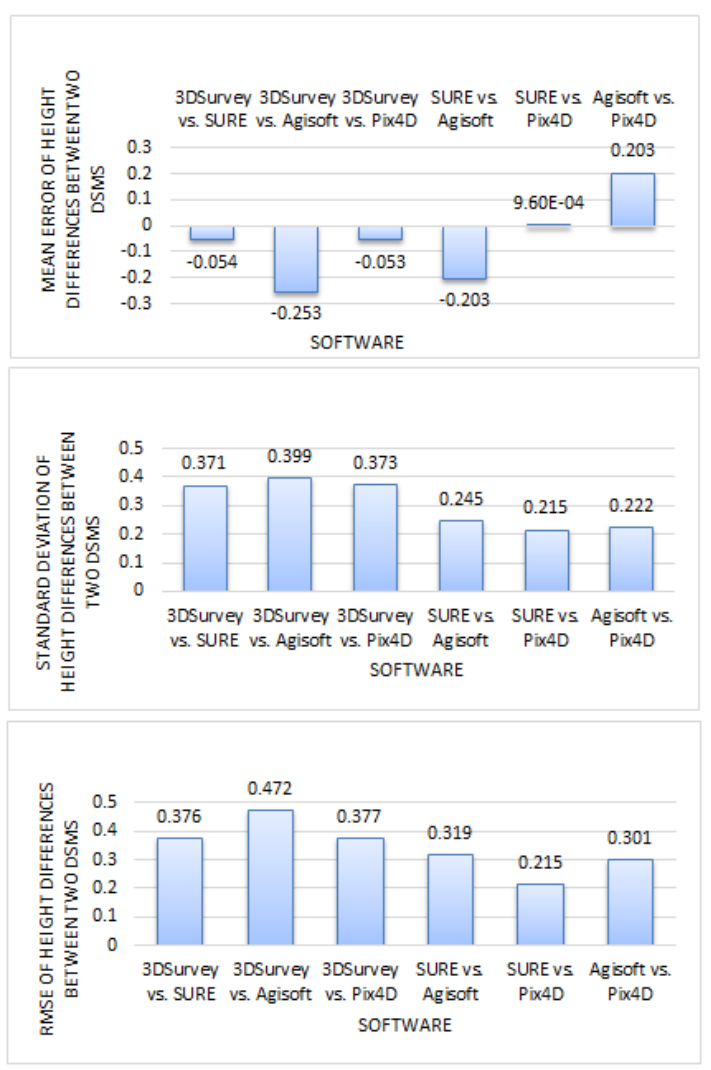

Figure 9. Standard measures [m] for accuracy assessment of two DSMs: the mean error (up), SD (middle), RMSE (down) 


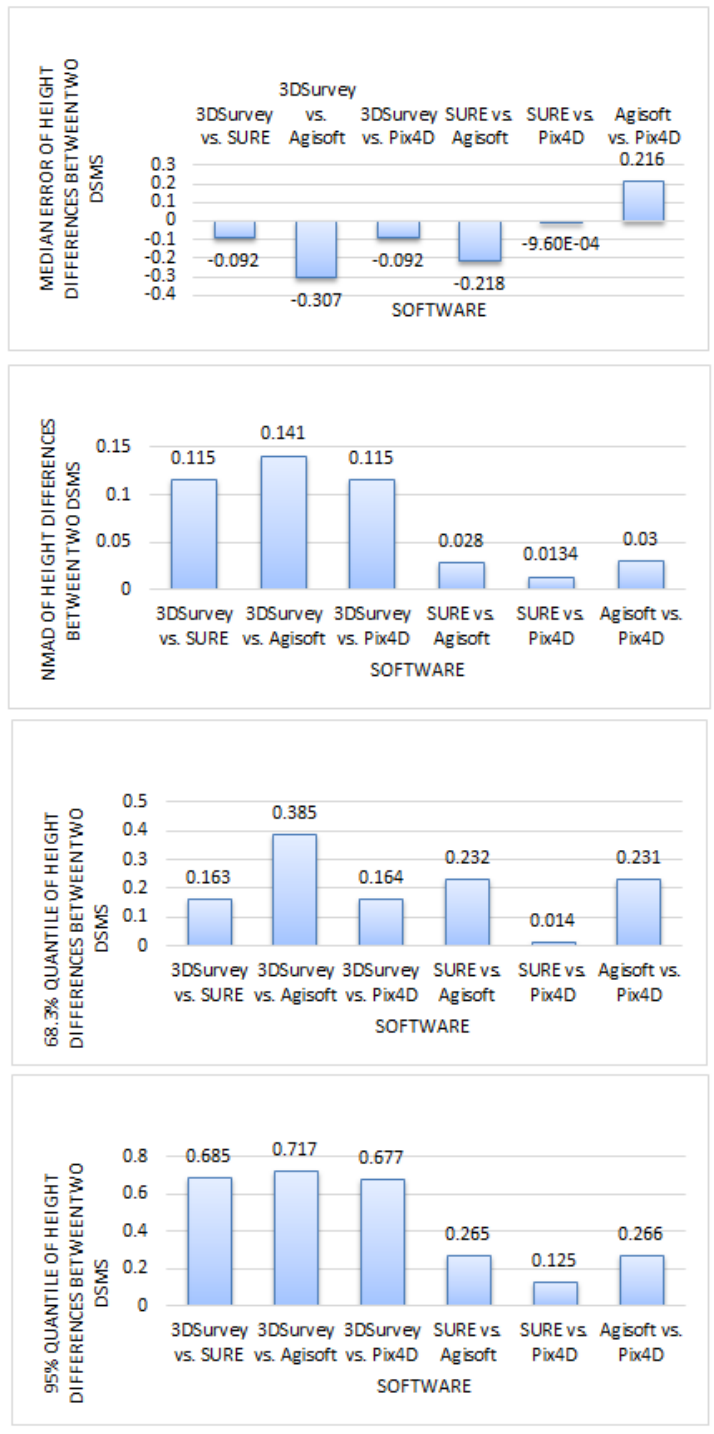

Figure 10. Robust measures for accuracy assessment of two DSMs: First: the median error $(\mathrm{m})$, Second: NMAD (m), Third: $68.3 \%$ quantile $(\mathrm{m})$, Forth: $95 \%$ quantile $(\mathrm{m})$

In order to have accurate and reliable assessments, NMAD and quantiles values can be used instead of standard statistical parameters (Höhle, 2009). As shown in Figure 10, The lowest value of NMAD $(0.01 \mathrm{~m})$ is for the height differences between Pix4Dmapper Pro's and SURE's DSMs, while the highest value is for the height differences between 3DSurvey's DSMs $(0.1 \mathrm{~m})$ and other three software. It indicates that the 3D models from Pix4Dmapper Pro, SURE and Agisoft Photoscan are similar to each other and are different from the 3DSurvey result.
Moreover, the error values in the $68.3 \%$ and $95 \%$ quantiles between 3DSurvey's DSMs and other three software indicate that about $27 \%$ of generated points by 3DSurvey lead to high elevation differences. In addition, $68.3 \%$ of height differences between Agisoft Photoscan's DSMs and Pix4Dmapper Pro's and SURE's DSMs is about $0.23 \mathrm{~m}$, while $95 \%$ of errors is about 0.26 $\mathrm{m}$.

In order to illustrate the distribution of height differences between DSMs, the Q-Q plots are used (Figure 11). As shown in Figure 11, the Q-Q plots related to 3DSurvey indicate a significant difference between the performances of 3DSurvey in point cloud generation against other three software.

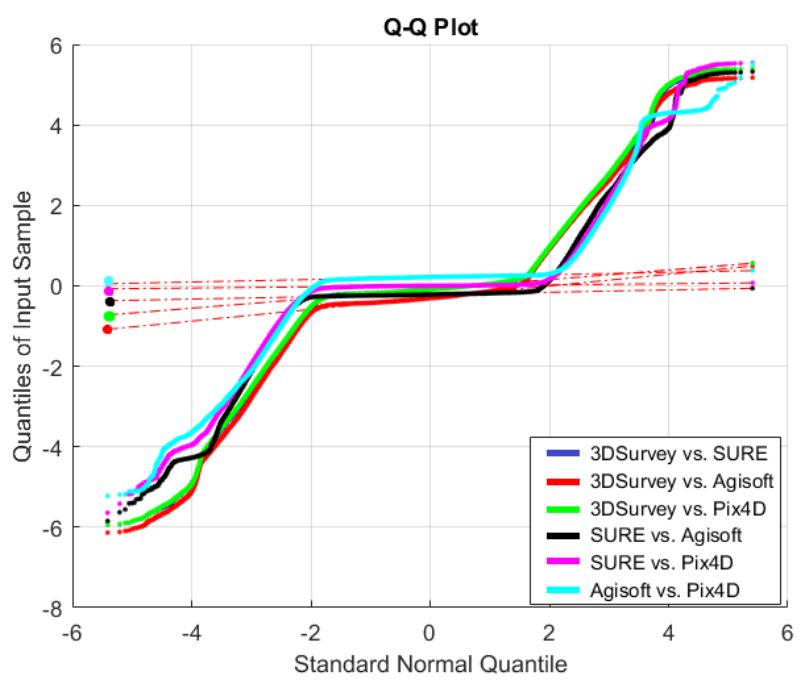

Figure 11. The Q-Q plots of height differences between two DSMs

Finally, other characteristics of software packages that they can considered for comparison, are as Table 3. For this analysis, the main components such as good documentation and report exporting, parameters controlling, user friendly, variety in output productions, independency, time and memory optimization can be voted as the score of 2 for very good and the score of 1 for good performance of different software packages. According to reported results of software comparisons, software with best performance could be selected based on the object of interest, geometric and visual accuracy of 3D reconstruction, resolution and scale of interest, software and hardware capabilities and the budget.

\begin{tabular}{|l|c|c|c|c|c|c|c|c|}
\hline Software & $\begin{array}{c}\text { Trial } \\
\text { license } \\
\text { validation } \\
\text { (day) }\end{array}$ & $\begin{array}{c}\text { Good } \\
\text { documentation } \\
\text { and report } \\
\text { exporting }\end{array}$ & $\begin{array}{c}\text { Parameters } \\
\text { controlling }\end{array}$ & $\begin{array}{c}\text { User } \\
\text { friendly }\end{array}$ & $\begin{array}{c}\text { Variety in } \\
\text { output } \\
\text { productions }\end{array}$ & Independency & $\begin{array}{c}\text { Time and } \\
\text { memory } \\
\text { optimization }\end{array}$ & $\begin{array}{c}\text { Sum } \\
\text { of } \\
\text { scores }\end{array}$ \\
\hline 3DSurvey & 15 & 1 & 1 & 1 & 1 & 2 & 1 & $\mathbf{7}$ \\
Agisoft & 30 & 2 & 2 & 2 & 2 & 2 & 1 & $\mathbf{1 1}$ \\
Pix4Dmapper & 7 & 2 & 2 & 2 & 2 & 2 & 1 & $\mathbf{1 2}$ \\
SURE & 14 & 1 & 1 & 2 & 2 & 1 & 1 & $\mathbf{8}$ \\
\hline
\end{tabular}

Table 3. Characteristics of software packages 


\section{CONCLUSION}

The result of this study demonstrate the capability of commercial photogrammetric software packages for automatic 3D reconstruction of different features using high resolution aerial images over a historical site. In this assessment, various visual and geometric metrics are used to measure the quality of generated point clouds as well as the performance of software. Despite existing the vertical shift in height values of Agisoft Photoscan's DSM, the 3D point clouds of Agisoft Photoscan, Pix4Dmapper Pro and SURE are more similar and 3DSurvey's point cloud has less accuracy and quality. However, the unsatisfying results could be improved by changing the parameter settings.

\section{ACKNOWLEDGEMENTS}

We thank four companies of Agisoft, Pix4D, nFrame and 3DSurvey for providing us the trial version of software packages.

\section{REFERENCES}

3DSurvey, 2017, http://www.3dsurvey.si (01 July 2017).

Agisoft Photoscan, 2017, http://www.agisoft.com (01 July 2017).

Alidoost, F., Arefi, H., 2015. An Image-Based Technique for 3D Building Reconstruction Using Multi-View UAV Images. In: The International Archives of the Photogrammetry, Remote Sensing and Spatial Information Sciences, Kish Island, Iran, Vol. XL-1/W5, pp. 43-46.

Bhandari, B., Oli, U., Pudasaini, U., Panta, N., 2015. Generation of High Resolution DSM Using UAV Images. Sofia, Bulgaria, In: FIG Working Week 2015, From the Wisdom of the Ages to the Challenges of the Modern World.

Burns, J.H.R., Delparte, D., 2017. Comparison of Commercial Structure-From-Motion Photogrammety Software used for Underwater Three-Dimensional Modeling of Coral Reef Environments. In: The International Archives of the Photogrammetry, Remote Sensing and Spatial Information Sciences, Nafplio, Greece, Vol. XLII-2/W3, pp. 127-131.

DJI, 2017, https://www.dji.com/phantom-4-pro (01 July 2017).

Haala, N., Cavegn, S., 2016. High Density Aerial Image Matching: State-of-The-Art and Future Prospects. In: The International Archives of the Photogrammetry, Remote Sensing and Spatial Information Sciences, Prague, Czech Republic, Vol. XLI-B4, pp. 625-630.

Haala, N., 2013. The Landscape of Dense Image Matching Algorithms. In: Photogrammetric Week '13, Wichmann/VDE Verlag, Berlin \& Offenbach.

Hirschmueller, H., 2005. Accurate and efficient stereo processing by semi-global matching and mutual information. In: Computer Vision and Pattern Recognition, IEEE Computer Society Conference on, Vol. 2, IEEE, pp. 807-814.

Höhle, J., Höhle, M., 2009. Accuracy assessment of digital elevation models by means of robust statistical methods. ISPRS Journal of Photogrammetry and Remote Sensing, 64(2009), pp. 398-406.
Niederheiser, R., Mokroš, M., Lange, J., Petschko H., Prasicek, G., Oude Elberink S.O., 2016. Deriving 3D Point Clouds from Terrestrial Photographs Comparison of Different Sensors and Software. In: The International Archives of the Photogrammetry, Remote Sensing and Spatial Information Sciences, Prague, Czech Republic, Vol. XLI-B5, pp. 685-692.

nFrame, 2017, http://www.nframes.com (01 July 2017).

Schwind, M., Starek, M.J., 2017. Assessing 3D Point Cloud Fidelity of UAS- SfMSoftware Solutions over Varying Terrain. In: 18th Annual JALBTCX Airborne Coastal Mapping and Charting Technical Workshop.

Pix4D, 2017, https://cloud.pix4d.com (01 July 2017).

Remondino, F., Grazia Spera, M., Nocerino, E., Menna, F., Nex, F., 2014. State of The Art in High Density Image Matching. Photogrammetric Record, 29(146), pp. 144-166.

Svensk, J., 2017. "Evaluation of Aerial Image Stereo Matching Methods for Forest Variable Estimation", Master of Science Thesis in Computer Vision, Department of Electrical Engineering, Linköping University.

SURE, Photogrammetric Surface Reconstruction from Imagery, Manual, ver. 12/5/14, www.nframe.com

Wikipedia, 2017, https://en.wikipedia.org/wiki/Harireh (01 July 2017)

Zečević, Z., Popović, T., Krstajić, B., 2017. Cloud Based Solution for Automatic Image, Mosaicking and Georeferencing. In: 22nd International Scientific-Professional Conference Information Technology, Zabljak, Montenegro, pp. 177-180. 